\title{
LITTLEWOOD-PALEY OPERATORS ON BMO
}

\author{
DOUGLAS S. KURTZ
}

\begin{abstract}
Two Littlewood-Paley operators, the area integral and the function $g_{\lambda}^{*}$, are considered as operators on functions of bounded mean oscillation. It is proved that the image of a BMO function under one of these operators is either equal to infinity almost everywhere or is in BMO.
\end{abstract}

Introduction. In [2], John and Nirenberg introduced the space BMO of functions of bounded mean oscillation. We say a locally integrable function $f$ is in $\operatorname{BMO}\left(\mathbf{R}^{n}\right)$ if there is a constant $C$ so that for all cubes $Q \subset \mathbf{R}^{n}$ there is a constant $c_{Q}$ depending only on $Q$ so that

$$
\frac{1}{|Q|} \int_{Q}\left|f(t)-c_{Q}\right| d t \leq C
$$

It is well known that $c_{Q}$ can always be chosen to be the average of $f$ over $Q$, $(1 /|Q|) \int_{Q} f(t) d t$. The smallest $C$ that satisfies the definition is called the BMO norm of $f$ and is denoted $\|f\|_{*}$. Identifying functions which differ by an additive constant makes BMO a Banach space. One interest in BMO is that many operators which are bounded on $L^{p}, 1<p<\infty$, but not on $L^{\infty}$, are bounded when considered as operators on BMO.

Wang $[\mathbf{7}]$ considered the $g$-function defined on BMO functions. Using a very simple proof, he proved

THEOREM. Let $f \in \operatorname{BMO}\left(\mathbf{R}^{n}\right)$. Either $g(f)(x)=\infty$ almost everywhere or $g(f)(x)<\infty$ almost everywhere and there is a constant $C$ depending only on $n$ such that $\|g(f)\|_{*} \leq C\|f\|_{*}$.

Thus, the $g$-function is not a bounded operator on all of BMO, but is bounded on the subset of functions whose $g$-function is finite almost everywhere. This leads one to consider whether the other Littlewood-Paley operators, the area integral $S(f)$ and the $g_{\lambda}^{*}$ function, satisfy similar results.

In [3], we prove estimates on the nonincreasing rearrangements of the three Littlewood-Paley operators. The techniques for all three are similar and the results yield information about the operators on spaces near $L^{\infty}$. Thus, it is not surprising that those techniques can be used to prove analogs of Wang's theorem. The main results of this paper are the following theorems.

THEOREM 1. Let $f \in \operatorname{BMO}\left(\mathbf{R}^{n}\right)$. Either $S(f)(x)=\infty$ almost everywhere or $S(f)(x)<\infty$ almost everywhere and there is a constant $C$ depending only on $n$ such that $\|S(f)\|_{*} \leq C\|f\|_{*}$.

Received by the editors February 19, 1986.

1980 Mathematics Subject Classification (1985 Revision). Primary 42B25.

Key words and phrases. Area integral, bounded mean oscillation. 
THEOREM 2. Let $f \in \operatorname{BMO}\left(\mathbf{R}^{n}\right)$ and $1<\lambda$. Either $g_{\lambda}^{*}(f)(x)=\infty$ almost everywhere or $g_{\lambda}^{*}(f)(x)<\infty$ almost everywhere and there is a constant $C$ depending on $n$ and $\lambda$ such that $\left\|g_{\lambda}^{*}(f)\right\|_{*} \leq C\|f\|_{*}$.

The proofs of these theorems use a single crude estimate on the gradient of the Poisson integral and an improvement of a result of Fefferman and Stein [1] concerning the integrability of BMO functions. Related results have been obtained recently by Rubio de Francia, Ruiz, and Torrea [4] and Shi and Torchinsky [5].

We note that Wang actually proved much more. There is a function $f \in L^{\infty}$ so that $g(f) \equiv \infty$ and an $h \in L^{\infty} \cap L^{2}$ so that $g(h)<\infty$ almost everywhere while $g(h) \notin L_{1}^{\infty}$. The pointwise relationships between these operators show his examples apply to the operators considered here. Further, there is an $f \in \mathrm{BMO} \cap L^{2}$ (so $g(f), S(f), g_{\lambda}^{*}(f)$ are finite almost everywhere) but $f \notin L^{\infty}$.

We assume all sets and functions are measurable. We use $\chi_{E}$ for the characteristic function of the measurable set $E$. If $Q$ is a cube in $\mathbf{R}^{n}, d Q$ stands for the cube concentric with $Q$ and having edge length $d$ times as long. We use $C$ to denote constants which may change from line to line. They depend on the indicated subscripts and never depend on $f$ or $x$.

I wish to thank Richard J. Bagby and Richard L. Wheeden for their helpful suggestions.

1. This section contains estimates of integrals of BMO functions and an outline of the proof of the main theorems. We begin by defining the Littlewood-Paley operators.

For $x \in \mathbf{R}^{n}$ and $y>0$, the Poisson kernel for the upper half-plane, $\mathbf{R}_{+}^{n+1}$, is $P(x, y)=c_{n} y\left(y^{2}+|x|^{2}\right)^{-(n+1) / 2}$. The Poisson integral of $f$ is

$$
f(x, y)=[f * P(\cdot, y)](x)=\int_{\mathbf{R}^{n}} f(z) P(x-z, y) d z .
$$

Since $P$ is harmonic on $\mathbf{R}_{+}^{n+1}, f(x, y)$ is harmonic. From the inequality

$$
|\nabla P(x, y)| \leq C_{n} y^{-1} P(x, y) \leq C_{n}(y+|x|)^{-(n+1)}
$$

we obtain

$$
|\nabla f(x, y)| \leq C_{n} \int_{\mathbf{R}^{n}}|f(z)|(y+|x-z|)^{-(n+1)} d z
$$

where

$$
\nabla f(x, y)=\left(\frac{\partial f}{\partial x_{1}}(x, y), \ldots, \frac{\partial f}{\partial x_{n}}(x, y), \frac{\partial f}{\partial y}(x, y)\right)
$$

is the full gradient of $f(x, y)$. It is surprising that this is the only estimate we use to handle the area integral and the Littlewood-Paley function. We need a better estimate for the $g$-function (see the end of $\S 2$ ).

Given a point $x \in \mathbf{R}^{n}$, define the right circular cone at $x$ by $\Gamma(x)=\{(z, y) \in$ $\left.\mathbf{R}_{+}^{n+1}:|z-x|<y\right\}$. The $g$-function $g(f)$, the area integral $S(f)$, and the LittlewoodPaley function $g_{\lambda}^{*}(f)$ are defined by

$$
\begin{gathered}
g(f)(x)=\left(\int_{0}^{\infty} y|\nabla f(x, y)|^{2} d y\right)^{1 / 2} \\
S(f)(x)=\left(\iint_{\Gamma(x)} y^{1-n}|\nabla f(z, y)|^{2} d z d y\right)^{1 / 2}
\end{gathered}
$$


and

$$
g_{\lambda}^{*}(f)(x)=\left(\iint_{\mathbf{R}_{+}^{n+1}}\left(\frac{y}{y+|x-z|}\right)^{\lambda n} y^{1-n}|\nabla f(z, y)|^{2} d z d y\right)^{1 / 2} .
$$

Let $Q$ be a cube centered at $x$ and having edge length $r$. In [1], Fefferman and Stein observe that an inequality of John and Nirenberg [2] implies

$$
\left(\frac{1}{|Q|} \int_{Q}\left|f(t)-f_{Q}\right|^{p} d t\right)^{1 / p} \leq C_{p}\|f\|_{*}
$$

for $f \in \operatorname{BMO}\left(\mathbf{R}^{n}\right)$. Further, they prove that for all $f \in \operatorname{BMO}\left(\mathbf{R}^{n}\right)$,

$$
\int_{\mathbf{R}^{n}} \frac{\left|f(t)-f_{Q}\right|}{|t-x|^{n+1}+r^{n+1}} d t \leq \frac{C}{r}\|f\|_{*} .
$$

This inequality implies the Poisson integral of $f$ and its gradient are well defined. An examination of their proof yields

LEMMA 1.1. Let $f \in \mathrm{BMO}\left(\mathbf{R}^{n}\right), \alpha>0$, and $p \geq 1$. Let $Q$ be a cube centered at $x$ and having edge length $r$. There is a constant $C$ depending on $n, \alpha$, and $p$ so that for $y>0$,

$$
\left(\int_{\mathbf{R}^{n}} \frac{\left|f(t)-f_{Q}\right|^{p}}{|t-x|^{n+\alpha}+y^{n+\alpha}} d t\right)^{1 / p} \leq C y^{-\alpha / p}(1+|\ln [y / r]|)\|f\|_{*} .
$$

Proof. Let $Q(k)$ be the cube concentric with $Q$ and having edge length $2^{k} r$. Using the observation above about $L^{p}$ averages of BMO functions and arguing by induction, one proves for $k \geq 1$ that

$$
\begin{aligned}
\left(\int_{Q(k)}\left|f(t)-f_{Q}\right|^{p} d t\right)^{1 / p} & \leq C_{p}(1+k)\left(1+2^{n / p}\right)\left(2^{k} r\right)^{n / p}\|f\|_{*} \\
& =C_{n, p}(1+k)\left(2^{k} r\right)^{n / p}\|f\|_{*} .
\end{aligned}
$$

Arguing as in [1], (1.2) implies

$$
\begin{gathered}
\left(\int_{\mathbf{R}^{n}} \frac{\left|f(t)-f_{Q}\right|^{p}}{|t-x|^{n+\alpha}+r^{n+\alpha}} d t\right)^{1 / p} \\
\leq C_{n, p} r^{-\alpha / p}\|f\|_{*}\left(1+\sum_{k=1}^{\infty}(1+k)^{p} 2^{-k \alpha}\right)^{1 / p} \\
\quad=C_{n, \alpha, p} r^{-\alpha / p}\|f\|_{*} .
\end{gathered}
$$

To complete the proof, let $R$ be the cube concentric with $Q$ and having edge length $y$. Then

$$
\begin{aligned}
\left(\int_{\mathbf{R}^{n}} \frac{\left|f(t)-f_{Q}\right|^{p}}{|t-x|^{n+\alpha}+y^{n+\alpha}} d t\right)^{1 / p} \leq & \left(\int_{\mathbf{R}^{n}} \frac{\left|f(t)-f_{R}\right|^{p}}{|t-x|^{n+\alpha}+y^{n+\alpha}} d t\right)^{1 / p} \\
& +\left|f_{R}-f_{Q}\right|\left(\int_{\mathbf{R}^{n}} \frac{d t}{|t-x|^{n+\alpha}+y^{n+\alpha}}\right)^{1 / p}
\end{aligned}
$$


By (1.3), the first term is bounded by $C_{n, \alpha, p} y^{-\alpha / p}\|f\|_{*}$. The integral in the second term equals $C_{n, \alpha, p} y^{-\alpha / p}$, so we need to prove that

$$
\left|f_{R}-f_{Q}\right| \leq C_{n}\left(1+\left|\log _{2}[y / r]\right|\right)\|f\|_{*} \text {. }
$$

Let $y>r$. Choose $k$ such that $2^{k} r \leq y<2^{k+1} r$. Then $2^{k+1} r / y \leq 2$ and $k \leq \log _{2}(y / r)$. By (1.2), with $p=1$ and $C=C_{n, 1}$,

$$
\begin{aligned}
\left|f_{R}-f_{Q}\right| & \leq y^{-n} \int_{R}\left|f(t)-f_{Q}\right| d t \leq y^{-n} \int_{Q(k+1)}\left|f(t)-f_{Q}\right| d t \\
& \leq C(2+k)\left(2^{k+1} r\right)^{n} y^{-n}\|f\|_{*} \leq C 2^{n}\left(2+\log _{2}[y / r]\right)\|f\|_{*} .
\end{aligned}
$$

If $y<r$, we reverse the roles of $y$ and $r$ to get the estimate above with $\log _{2}[r / y]=$ $\left|\log _{2}[y / r]\right|$. Since $1+|\ln [y / r]|$ is equivalent to $2+\left|\log _{2}[y / r]\right|,(1.4)$ is proved which completes the proof of the lemma.

Let $T f$ be one of the Littlewood-Paley operators. The main idea of the proof of Theorems 1 and 2 is to reduce the study of $T f$ to that of $T\left(\left[f-f_{Q}\right] \chi_{C Q}\right)$, where $C Q$ is the complement of $Q$. This is the proof Wang uses in [7]. For the sake of completeness, we sketch the proof.

Suppose $T f \neq \infty$ almost everywhere. Then $E=\{x: T f(x)<\infty\}$ has positive measure. Let $\underline{x}$ be a point of density of $E$. Let $Q$ be any cube centered at $\underline{x}$ and set $f_{Q}=(1 /|Q|) \int_{Q} f(t) d t$. Write $f$ as

$$
f(x)=f_{Q}+\left[f(x)-f_{Q}\right] \chi_{Q}(x)+\left[f(x)-f_{Q}\right] \chi_{C Q}(x)=f_{Q}+g_{Q}(x)+h_{Q}(x) .
$$

Since $f_{Q}$ is a constant, $T f_{Q}$ is identically 0 . Thus, $T f_{Q}$ is in BMO with BMO norm equal to 0 . Therefore, $T f \leq T g_{Q}+T h_{Q}$ and $T h_{Q} \leq T g_{Q}+T f$.

Since $f \in \operatorname{BMO}\left(\mathbf{R}^{n}\right)$,

$$
\left\|g_{Q}\right\|_{2}=\left(\int_{Q}\left|f(t)-f_{Q}\right|^{2} d t\right)^{1 / 2} \leq C|Q|^{1 / 2}\|f\|_{*}
$$

and $g_{Q} \in L^{2}$. Thus, $T g_{Q}$ is finite almost everywhere (see [6, pp. 89-91]). Therefore, $T f(x)<\infty$ at almost every point such that $T h_{Q}(x)<\infty$.

Let $d=d_{n}<1$. Since $\underline{x}$ is a point of density of $E$ and $T g_{Q}$ is finite almost everywhere, there is a point $x^{\prime}$ in $d Q$ (possibly $\underline{x}$ ) so that $T f\left(x^{\prime}\right), T g_{Q}\left(x^{\prime}\right)$, and (therefore) $T h_{Q}\left(x^{\prime}\right)$ are finite. Assume we have proved for sufficiently small $d$, depending only on $n$, that there is a constant $C$ depending only on $n$ (and $\lambda$ ) so that for all $x \in d Q$ :

(i) $T h_{Q}\left(x^{\prime}\right)<\infty \Rightarrow T h_{Q}(x)<\infty$,

(ii) $\left|T h_{Q}(x)-T h_{Q}\left(x^{\prime}\right)\right| \leq C\|f\|_{*}$.

Fix a cube $Q$ centered at $\underline{x}$. As above, there is an $x^{\prime} \in d Q$ so that $T h_{Q}\left(x^{\prime}\right)<\infty$. By (i), $T h_{Q}(x)$ and, thus, $T f(x)$ is finite almost everywhere in $d Q$. Considering only cubes centered at $\underline{x}$ with edge length equal to a positive integer shows $T f$ is finite almost everywhere.

We have left to prove that $\|T f\|_{*} \leq C\|f\|_{*}$. Let $Q^{\prime} \subset \mathbf{R}^{n}$ be any cube and set $Q=(1 / d) Q^{\prime}$ (so that $Q^{\prime}=d Q$ ). Choose a point $x^{\prime} \in d Q$ so that $T h_{Q}\left(x^{\prime}\right)$ is finite. 
Then, by (1.5) and (ii),

$$
\begin{aligned}
& \frac{1}{\left|Q^{\prime}\right|} \int_{Q^{\prime}}\left|T f(x)-T h_{Q}\left(x^{\prime}\right)\right| d x \\
& \quad=\frac{1}{\left|Q^{\prime}\right|} \int_{Q^{\prime}}\left|T\left(g_{Q}+h_{Q}\right)(x)-T h_{Q}(x)+T h_{Q}(x)-T h_{Q}\left(x^{\prime}\right)\right| d x \\
& \quad \leq \frac{1}{\left|Q^{\prime}\right|} \int_{Q^{\prime}}\left|T g_{Q}(x)\right| d x+\frac{1}{\left|Q^{\prime}\right|} \int_{Q^{\prime}}\left|T h_{Q}(x)-T h_{Q}\left(x^{\prime}\right)\right| d x \leq C\|f\|_{*} .
\end{aligned}
$$

Since any constant $c_{Q^{\prime}}$ which depends only on the cube can be used in the definition of $\mathrm{BMO}$ and $Q^{\prime}$ is arbitrary, the proof is complete.

Theorems 1 and 2 are proved modulo the results of the next section.

2. We now prove the lemmas needed to justify (i) and (ii). Recall from the previous section that given a function $f$ and a cube $Q$, we define

$$
h_{Q}(x)=\left[f(x)-f_{Q}\right] \chi_{C Q}(x) .
$$

LEMMA 2.1. Suppose $f \in \operatorname{BMO}\left(\mathbf{R}^{n}\right)$. Let $Q$ be a cube with center $\underline{x}$ and edge length $r$. Set $d=1 /(8 \sqrt{n})$. Suppose there is an $x^{\prime} \in d Q$ so that $S\left(h_{Q}\right)\left(x^{\prime}\right)<$ $\infty$. There is a constant $C$, depending only on $n$, such that $S\left(h_{Q}\right)(x)<\infty$ and $\left|S\left(h_{Q}\right)(x)-S\left(h_{Q}\right)\left(x^{\prime}\right)\right| \leq C\|f\|_{*}$ for all $x \in d Q$.

ProOF. Fix $x \in d Q$. Set $\Gamma^{-}(x)=\{(z, y) \in \Gamma(x): y \leq d r\}$ and $\Gamma^{+}(x)=$ $\{(z, y) \in \Gamma(x): y>d r\}$. Then, $S\left(h_{Q}\right)(x)$ is bounded by the sum of

$$
S^{-}=\left(\iint_{\Gamma^{-}(x)} y^{1-n}\left|\nabla h_{Q}(z, y)\right|^{2} d z d y\right)^{1 / 2}
$$

and

$$
S^{+}=\left(\iint_{\Gamma^{+}(x)} y^{1-n}\left|\nabla h_{Q}(z, y)\right|^{2} d z d y\right)^{1 / 2}
$$

By (1.1),

$$
S^{-} \leq C_{n}\left(\iint_{\Gamma^{-}(x)} y^{1-n}\left(\int_{C Q} \frac{\left|f(t)-f_{Q}\right|}{|t-z|^{n+1}+y^{n+1}} d t\right)^{2} d z d y\right)^{1 / 2}
$$

Since $(z, y) \in \Gamma^{-}(x), x \in d Q$, and $t \notin Q$,

$$
|\underline{x}-t| \geq r / 2, \quad|x-\underline{x}| \leq r / 8, \quad \text { and } \quad|x-z|<y<r / 8 .
$$

Thus, $|t-z|>|\underline{x}-t|-|x-\underline{x}|-|x-z|>|\underline{x}-t|-r / 4>|\underline{x}-t| / 2$. Applying Lemma 1.1 ,

$$
\begin{aligned}
S^{-} & \leq C_{n}\left(\iint_{\Gamma^{-}(x)} y^{1-n}\left(\int_{C Q} \frac{\left|f(t)-f_{Q}\right|}{|t-\underline{x}|^{n+1}+r^{n+1}} d t\right)^{2} d z d y\right)^{1 / 2} \\
& \leq C_{n}\left(\int_{0}^{d r} \int_{\{|z-x|<y\}} y^{1-n} r^{-2}\|f\|_{*}^{2} d z d y\right)^{1 / 2} \\
& \leq C_{n}\|f\|_{*}\left(\int_{0}^{r} y r^{-2} d y\right)^{1 / 2}=C_{n}\|f\|_{*} .
\end{aligned}
$$


For $S^{+}$we have

$$
\begin{aligned}
S^{+}= & \left(\iint_{\Gamma^{+}(x)} y^{1-n}\left|\nabla h_{Q}(z, y)\right|^{2} d z d y\right)^{1 / 2} \\
\leq & \left(\iint_{\Gamma^{+}(x) \cap \Gamma\left(x^{\prime}\right)} y^{1-n}\left|\nabla h_{Q}\right|^{2} d z d y\right)^{1 / 2} \\
& +\left(\iint_{\Gamma^{+}(x) \backslash \Gamma\left(x^{\prime}\right)} y^{1-n}\left|\nabla h_{Q}\right|^{2} d z d y\right)^{1 / 2}
\end{aligned}
$$

The first term is bounded by $S\left(h_{Q}\right)\left(x^{\prime}\right)$. Suppose $(z, y) \in \Gamma^{+}(x)$ and $t \notin Q$. If $|t-\underline{x}| \leq 20 \sqrt{n} y$, then

$$
|z-t| \leq|z-x|+|x-\underline{x}|+|\underline{x}-t| \leq y+r+20 \sqrt{n} y \leq 30 \sqrt{n} y .
$$

If $|t-\underline{x}|>20 \sqrt{n} y$, we have

$$
|t-\underline{x}| / 2<|t-\underline{x}|-|z-\underline{x}| \leq|z-t| \leq|t-\underline{x}|+|z-\underline{x}|<2|t-\underline{x}| .
$$

Therefore, we always have that $|\underline{x}-t|+y$ is equivalent to $|z-t|+y$. Using (1.1) and Lemma 1.1, the second term is bounded by

$$
\begin{gathered}
C_{n}\left(\iint_{\Gamma^{+}(x) \backslash \Gamma\left(x^{\prime}\right)} y^{1-n}\left(\int_{C Q} \frac{\left|f(t)-f_{Q}\right|}{|t-z|^{n+1}+y^{n+1}} d t\right)^{2} d z d y\right)^{1 / 2} \\
\quad \leq C_{n}\left(\iint_{\Gamma^{+}(x) \backslash \Gamma\left(x^{\prime}\right)} y^{1-n}\left(\int_{C Q} \frac{\left|f(t)-f_{Q}\right|}{|t-\underline{x}|^{n+1}+y^{n+1}} d t\right)^{2} d z d y\right)^{1 / 2} \\
\quad \leq C_{n}\left(\iint_{\Gamma^{+}(x) \backslash \Gamma\left(x^{\prime}\right)} y^{1-n} y^{-2}\left(1+\left|\ln \left[\frac{y}{r}\right]\right|\right)^{2}\|f\|_{*}^{2} d z d y\right)^{1 / 2}
\end{gathered}
$$

For fixed $y_{0}$, we have that

$$
\begin{gathered}
\left\{\Gamma^{+}(x) \backslash \Gamma\left(x^{\prime}\right)\right\} \cap\left\{y=y_{0}\right\}=\left\{z:|z-x|<y_{0},\left|z-x^{\prime}\right|>y_{0}\right\} \\
\subset\left\{z: y_{0}-\left|x-x^{\prime}\right|<|z-x|<y_{0}\right\} .
\end{gathered}
$$

Simple estimates show that the measure of this last set is bounded by $C_{n} y_{0}^{n-1}\left|x-x^{\prime}\right|$. Since $\left|x-x^{\prime}\right| \leq r$, performing the $z$ integration yields

$$
\begin{aligned}
S^{+} & \leq S\left(h_{Q}\right)\left(x^{\prime}\right)+C_{n}\left(\int_{d r}^{\infty}\left|x-x^{\prime}\right| y^{n-1} y^{-1-n}\left(1+\left|\ln \left[\frac{y}{r}\right]\right|\right)^{2}\|f\|_{*}^{2} d y\right)^{1 / 2} \\
& \leq S\left(h_{Q}\right)\left(x^{\prime}\right)+C_{n}\|f\|_{*}\left(\int_{d r}^{\infty} r y^{-2}\left(1+\left|\ln \left[\frac{y}{r}\right]\right|\right)^{2} d y\right)^{1 / 2} \\
& \leq S\left(h_{Q}\right)\left(x^{\prime}\right)+C_{n}\|f\|_{*}\left(\int_{d}^{\infty} t^{-2}(1+|\ln [t]|)^{2} d t\right)^{1 / 2}=S h_{Q}\left(x^{\prime}\right)+C_{n}\|f\|_{*} .
\end{aligned}
$$

Combining these estimates shows that

$$
S\left(h_{Q}\right)(x) \leq S\left(h_{Q}\right)\left(x^{\prime}\right)+C_{n}\|f\|_{*}
$$


Thus, $S\left(h_{Q}\right)(x)$ is finite. Reversing the roles of $x$ and $x^{\prime}$ we get

$$
\left|S\left(h_{Q}\right)(x)-S\left(h_{Q}\right)\left(x^{\prime}\right)\right| \leq C_{n}\|f\|_{*}
$$

This completes the proof of Lemma 2.1 .

The following result is the analog of Lemma 2.1 for the $g_{\lambda}^{*}$ function.

LEMMA 2.2. Suppose $f \in \mathrm{BMO}\left(\mathbf{R}^{n}\right)$ and $1<\lambda$. Let $Q$ be a cube with center $\underline{x}$ and edge length $r$. Set $d=1 /(8 \sqrt{n})$. Suppose there is an $x^{\prime} \in d Q$ so that $g_{\lambda}^{*}\left(h_{Q}\right)\left(x^{\prime}\right)<\infty$. There is a constant $C$, depending on $n$ and $\lambda$, such that $g_{\lambda}^{*}\left(h_{Q}\right)(x)<\infty$ and $\left|g_{\lambda}^{*}\left(h_{Q}\right)(x)-g_{\lambda}^{*}\left(h_{Q}\right)(\underline{x})\right| \leq C\|f\|_{*}$ for all $x \in d Q$.

PRoOF. Let $J(k)=\left\{(z, y) \in \mathbf{R}_{+}^{n+1}:|z-\underline{x}|<2^{k-2} r\right.$ and $\left.0<y<2^{k-2} r\right\}$ for $k \geq 0$. Fix $x \in d Q$. Bound $g_{\lambda}^{*}\left(h_{Q}\right)(x)$ by the sum of

$$
G^{-}=\left(\iint_{J(0)}\left(\frac{y}{y+|x-z|}\right)^{\lambda n} y^{1-n}\left|\nabla h_{Q}(z, y)\right|^{2} d z d y\right)^{1 / 2}
$$

and

$$
G^{+}\left(\iint_{\mathbf{R}_{+}^{n+1} \backslash J(0)}\left(\frac{y}{y+|x-z|}\right)^{\lambda n} y^{1-n}\left|\nabla h_{Q}(z, y)\right|^{2} d z d y\right)^{1 / 2} .
$$

As before, if $(z, y) \in J(0)$ and $t \notin Q$, then $|z-t|>|\underline{x}-t| / 2>r / 4>y$. By (1.1) and Lemma 1.1,

$$
\begin{aligned}
G^{-} & \leq C_{n}\left(\iint_{J(0)}\left(\frac{y}{y+|x-z|}\right)^{\lambda n} y^{1-n}\left(\int_{C Q} \frac{\left|f(t)-f_{Q}\right|}{|t-z|^{n+1}+y^{n+1}} d t\right)^{2} d z d y\right)^{1 / 2} \\
& \leq C_{n}\left(\iint_{J(0)}\left(\frac{y}{y+|x-z|}\right)^{\lambda n} y^{1-n}\left(\int_{C Q} \frac{\left|f(t)-f_{Q}\right|}{|t-\underline{x}|^{n+1}+r^{n+1}} d t\right)^{2} d z d y\right)^{1 / 2} \\
& \leq C_{n}\left(\int_{0}^{r} \int_{\{|z-x|<r\}}\left(\frac{y}{y+|x-z|}\right)^{\lambda n} y^{1-n} r^{-2}\|f\|_{*}^{2} d z d y\right)^{1 / 2} .
\end{aligned}
$$

The change of variables $t=(x-z) / y$ and the fact that $\lambda n>n$ imply

$$
G^{-} \leq C_{n}\|f\|_{*}\left(\int_{0}^{r} \int_{\{|t|<r / y\}}\left(\frac{1}{1+|t|}\right)^{\lambda n} y r^{-2} d t d y\right)^{1 / 2}=C_{n}\|f\|_{*} .
$$

To estimate $G^{+}$, observe that

$$
\begin{aligned}
& G^{+}=\left(\iint_{\mathbf{R}_{+}^{n+1} \backslash J(0)}\left(\frac{y}{y+|x-z|}\right)^{\lambda n} y^{1-n}\left|\nabla h_{Q}(z, y)\right|^{2} d z d y\right)^{1 / 2} \\
& \leq\left(\iint_{\mathbf{R}_{+}^{n+1} \backslash J(0)}\left(\frac{y}{y+\left|x^{\prime}-z\right|}\right)^{\lambda n} y^{1-n}\left|\nabla h_{Q}(z, y)\right|^{2} d z d y\right)^{1 / 2} \\
&+\left(\iint_{\mathbf{R}_{+}^{n+1} \backslash J(0)}\left|\left(\frac{1}{y+|x-z|}\right)^{\lambda n}-\left(\frac{1}{y+\left|x^{\prime}-z\right|}\right)^{\lambda n}\right|\right. \\
&\left.\times y^{\lambda n+1-n}\left|\nabla h_{Q}(z, y)\right|^{2} d z d y\right)^{1 / 2} .
\end{aligned}
$$

The first term is bounded by $g_{\lambda}^{*}\left(h_{Q}\right)\left(x^{\prime}\right)$. 
Call the second term $\tau$. If $(z, y) \notin J_{0},|z-\underline{x}|+y$ is equivalent to both $\left|z-x^{\prime}\right|+y$ and $|z-x|+y$. By the Mean Value Theorem,

$$
\begin{aligned}
& \left|\left(\frac{1}{y+|x-z|}\right)^{\lambda n}-\left(\frac{1}{y+\left|x^{\prime}-z\right|}\right)^{\lambda n}\right| \\
& \quad \leq C_{n, \lambda} \frac{\left|x-x^{\prime}\right|}{(y+|\underline{x}-z|)^{\lambda n+1}} \leq \frac{C_{n, \lambda} r}{(y+|\underline{x}-z|)^{\lambda n+1}} .
\end{aligned}
$$

If $(z, y) \in J(k) \backslash J(k-1)$, then $|z-\underline{x}|+y$ is equivalent to $2^{k} r$. Therefore,

$$
\begin{aligned}
\tau & \leq C_{n, \lambda}\left(\iint_{\mathbf{R}_{+}^{n+1} \backslash J(0)} r(y+|\underline{x}-z|)^{-\lambda n-1} y^{\lambda n+1-n}\left|\nabla h_{Q}(z, y)\right|^{2} d z d y\right)^{1 / 2} \\
& \leq C_{n, \lambda}\left(\sum_{k=1}^{\infty} \iint_{J(k) \backslash J(k-1)} r(y+|\underline{x}-z|)^{-\lambda n-1} y^{\lambda n+1-n}\left|\nabla h_{Q}(z, y)\right|^{2} d z d y\right)^{1 / 2} \\
& \leq C_{n, \lambda}\left(\sum_{k=1}^{\infty} 2^{-k}\left(2^{k} r\right)^{-\lambda n} \iint_{J(k) \backslash J(k-1)} y^{\lambda n+1-n}\left|\nabla h_{Q}(z, y)\right|^{2} d z d y\right)^{1 / 2}
\end{aligned}
$$

Without loss of generality, we may assume that $1<\lambda \leq \Lambda<2$, since $y<2^{k} r$ and $\lambda>\Lambda$ imply that $\left(y / 2^{k} r\right)^{\lambda n} \leq\left(y / 2^{k} r\right)^{\Lambda n}$. It remains to prove that for $1<\lambda \leq$ $\Lambda<2$,

$$
\iint_{J(k)} y^{\lambda n+1-n}\left|\nabla h_{Q}(z, y)\right|^{2} d z d y \leq C_{n, \lambda}(1+k)^{2}\left(2^{k} r\right)^{\lambda n}\|f\|_{*}^{2} .
$$

Define $Q(k)$ as in the proof of Lemma 1.1. By (1.1), we have

$$
\begin{aligned}
\iint_{J(k)} y^{\lambda n+1-n}\left|\nabla h_{Q}(z, y)\right|^{2} d z d y & \\
\leq & C_{n} \iint_{J(k)} y^{\lambda n+1-n}\left(\int_{C Q} \frac{\left|f(t)-f_{Q}\right|}{|t-z|^{n+1}+y^{n+1}} d t\right)^{2} d z d y \\
\leq & C_{n} \iint_{J(k)} y^{\lambda n+1-n}\left(\int_{C Q(k+1)} \frac{\left|f(t)-f_{Q}\right|}{|t-z|^{n+1}+y^{n+1}} d t\right)^{2} d z d y \\
& +C_{n} \iint_{J(k)} y^{\lambda n+1-n}\left(\int_{Q(k+1) \backslash Q} \frac{\left|f(t)-f_{Q}\right|}{|t-z|^{n+1}+y^{n+1}} d t\right)^{2} d z d y \\
= & A_{k}+B_{k} .
\end{aligned}
$$

Since $(z, y) \in J(k)$, if $t \notin Q(k+1)$ then $|z-t|+y$ is equivalent to $|\underline{x}-t|+y$, and so it is equivalent to $|\underline{x}-t|+2^{k} r$. Thus,

$$
A_{k} \leq C_{n} \iint_{J(k)} y^{\lambda n+1-n}\left(\int_{C Q(k+1)} \frac{\left|f(t)-f_{Q}\right|}{|t-\underline{x}|^{n+1}+\left(2^{k} r\right)^{n+1}} d t\right)^{2} d z d y
$$


By Lemma 1.1 and a change of variables,

$$
\begin{aligned}
A_{k} & \leq C_{n} \int_{0}^{2^{k} r} \int_{\left\{|z| \leq 2^{k} r\right\}} y^{\lambda n+1-n}\left(2^{k} r\right)^{-2}(1+k)^{2}\|f\|_{*}^{2} d z d y \\
& \leq C_{n, \lambda}\left(2^{k} r\right)^{\lambda n+2-n+n-2}(1+k)^{2}\|f\|_{*}^{2}=C_{n, \lambda}\left(2^{k} r\right)^{\lambda n}(1+k)^{2}\|f\|_{*}^{2} .
\end{aligned}
$$

Arguing as in the proof of inequality (3.4) of [3], to estimate $B_{k}$ change the order of integration, apply Minkowski's integral inequality to the inner two integrals, and expand the domains of integration to get

$$
\begin{aligned}
B_{k} \leq C_{n} \int_{\left\{|z-\underline{x}|<2^{k} r\right\}} & {\left[\int_{0}^{2^{k} r} y^{\lambda n+1-n}\left(\int_{Q(k+1) \backslash Q} \frac{\left|f(t)-f_{Q}\right|}{|t-z|^{n+1}+y^{n+1}} d t\right)^{2} d y\right] d z } \\
\leq C_{n} \int_{\mathbf{R}^{n}}\left[\int_{Q(k+1) \backslash Q}\left|f(t)-f_{Q}\right|\right. & \left.\times\left(\int_{0}^{\infty} \frac{y^{\lambda n+1-n}}{|t-z|^{2(n+1)}+y^{2(n+1)}} d y\right)^{1 / 2} d t\right]^{2} d z .
\end{aligned}
$$

Integrating first between 0 and $|t-z|$, then between $|t-z|$ and $\infty$, the innermost integral is bounded by a constant times $|t-z|^{-3 n+\lambda n}$. Write $-3 n+\lambda n=$ $-2 n+(\lambda-1) n$. Inserting this estimate and using the Hardy-Littlewood-Sobolev theorem for fractional integration $[6$, p. 119] with $\gamma=[(\lambda-1) / 2] n, q=2$, and $1 / p=\frac{1}{2}+(\lambda-1) / 2=\lambda / 2$, we obtain

$$
\begin{aligned}
B_{k} & \leq C_{n, \lambda} \int_{\mathbf{R}^{n}}\left(\int_{Q(k+1) \backslash Q}\left|f(t)-f_{Q}\right||z-t|^{-n+n(\lambda-1) / 2} d t\right)^{2} d z \\
& \leq C_{n, \lambda}\left(\int_{Q(k+1) \backslash Q}\left|f(t)-f_{Q}\right|^{2 / \lambda} d t\right)^{\lambda} .
\end{aligned}
$$

Applying (1.2) with $p=2 / \lambda$,

$$
B_{k} \leq C_{n, \lambda}(2+k)^{2}\left(2^{k+1} r\right)^{\lambda n}\|f\|_{*}^{2} .
$$

Combining the estimates for $A_{k}$ and $B_{k}$ completes the proof of (2.1). This implies

$$
\tau \leq C_{n, \lambda}\|f\|_{*}\left(\sum_{k=1}^{\infty}(1+k)^{2} 2^{-k}\right)^{1 / 2}=C_{n, \lambda}\|f\|_{*},
$$

so that $G^{+} \leq g_{\lambda}^{*}\left(h_{Q}\right)\left(x^{\prime}\right)+C_{n, \lambda}\|f\|_{*}$. As in the previous proof, it now follows that $g_{\lambda}^{*}\left(h_{Q}\right)(x)<\infty$ and $\left|g_{\lambda}^{*}\left(h_{Q}\right)(x)-g_{\lambda}^{*}\left(h_{Q}\right)\left(x^{\prime}\right)\right| \leq C_{n, \lambda}\|f\|_{*}$.

We note that estimates similar to those of [3] can be used to prove Wang's $g$ function result. In [7], the crux of the proof is to estimate $\left|\nabla h_{Q}(x, y)-\nabla h_{Q}(\underline{x}, y)\right|^{2}$ (our notation). This is similar to the expression in the conclusion of Proposition 1.2 of [3], which is not a sufficiently sharp result. However, the proof of the proposition actually yields the stronger result:

Suppose $|x-\underline{x}| \leq y$. There is a constant $C_{n}$ so that

$$
\left.|| \nabla f(x, y)\right|^{2}-|\nabla f(\underline{x}, y)|^{2}\left|\leq C_{n}\right| x-\left.\underline{x}\left|y^{-3} \sup \right| f(\xi, \eta)\right|^{2},
$$


the sup is taken over all $(\xi, \eta)$ with $|\xi-\underline{x}| \leq 6 y$ and $y / 2 \leq \eta \leq 2 y$.

This estimate, the definition of the Poisson integral of $f$, and Lemma 1.1 show that for $y>r \geq|x-\underline{x}|$

$$
\left.|| \nabla h_{Q}(x, y)\right|^{2}-\left|\nabla h_{Q}(\underline{x}, y)\right|^{2}\left|\leq C_{n}\right| x-\underline{x} \mid y^{-3}(1+\log [y / r])^{2}\|f\|_{*}^{2} .
$$

Using the proof of the preceding lemmas, this is enough to prove (i) and (ii) for the $g$-function and, hence, the BMO result.

\section{REFERENCES}

1. C. Fefferman and E. M. Stein, $H^{p}$ spaces of several variables, Acta Math. 129 (1972), 137-193.

2. F. John and L. Nirenberg, On functions of bounded mean oscillation, Comm. Pure Appl. Math. 14 (1961), 415-426.

3. D. S. Kurtz, Rearrangement inequalities for Littlewood-Paley operators, Math. Nachr. (to appear).

4. J. L. Rubio de Francia, F. J. Ruiz and J. L. Torrea, Les opérateurs de Calderón-Zygmund vectoriels, C. R. Acad. Sci. Paris 297 (1983), 477-480.

5. X. Shi and A. Torchinsky (oral communication).

6. E. M. Stein, Singular integrals and differentiability properties of functions, Princeton Univ. Press, Princeton, N.J., 1970.

7. S. Wang, Some properties of the Littlewood-Paley g-function, Contemp. Math., Vol. 42, Amer. Math. Soc., Providence, R. I., 1985, pp. 191-202.

Department of Mathematical Sciences, New Mexico State University, Las CRUCES, NeW MEXICO 88003 